tyrosine kinase (Btk) is essential for human B cell tolerance. J. Exp. Med. 200:927-934.

11. Logtenberg, T., Young, F.M., van Es, J.H., GmeligMeyling, F.H., and Alt, F.W. 1989. Autoantibodies encoded by the most JH-proximal human immunoglobulin heavy chain variable region gene. J. Exp. Med. 170:1347-1355.

12. Ochsenbein, A.F., and Zinkernagel, R.M. 2000. Natural antibodies and complement link innate and acquired immunity. Immunol. Today. 21:624-630.

13. Pascual, V., et al. 1991. Nucleotide sequence analysis of the $\mathrm{V}$ regions of two IgM cold agglutinins. Evidence that the VH4-21 gene segment is responsible for the major cross-reactive idiotype. J. Immunol. 146:4385-4391.

14. Zhang, M., et al. 2006. Identification of the target self-antigens in reperfusion injury. J. Exp. Med. 203:141-152

15. Ray, S.K., Putterman, C., and Diamond, B. 1996. Pathogenic autoantibodies are routinely generated during the response to foreign antigen: a paradigm for autoimmune disease. Proc. Natl. Acad. Sci.U.S. A. 93:2019-2024.

16. Pugh-Bernard, A.E., et al. 2001. Regulation of inherently autoreactive VH4-34 B cells in the maintenance of human $\mathrm{B}$ cell tolerance.
J. Clin. Invest. 108:1061-1070. doi:10.1172/ JCI200112462.

17. Van Vollenhoven, R.F., et al. 1999. VH4-34 encoded antibodies in systemic lupus erythematosus: a specific diagnostic marker that correlates with clinical disease characteristics. J. Rheumatol. 26:1727-1733

18. Akira, S., Takeda, K., and Kaisho, T. 2001. Tolllike receptors: critical proteins linking innate and acquired immunity. Nat. Immunol. 2:675-680.

19. Marshak-Rothstein, A. 2006. Toll-like receptors in systemic autoimmune disease. Nat. Rev. Immunol. 6:823-835.

\title{
Scavenger receptors clear the air
}

\author{
Edward M. Postlethwait
}

\author{
Department of Environmental Health Sciences, University of Alabama at Birmingham, Birmingham, Alabama, USA.
}

\begin{abstract}
Inhaled environmental oxidants, such as ozone and particulates, have been variably linked to epithelial injury, inflammation, and perturbations in lung development, growth, and function. Reactions between ozone and lung surface lipids likely account for exposure-related pathophysiologic sequelae. In this issue of the JCI, Dahl et al. document a previously unrecognized pulmonary defense against inhaled oxidants in mice: macrophage scavenger receptors (SRs) bind proinflammatory oxidized lipids, thereby decreasing pulmonary inflammation (see the related article beginning on page 757 ). The study adds to our knowledge of diverse lung oxidative processes and identifies a potential regulatory mechanism governing pulmonary inflammation. Further investigations to elucidate more precise mechanisms and to determine the influence of SRs on airway epithelial injury, repair, and remodeling are warranted.
\end{abstract}

\section{Scavenger receptors}

The term scavenger receptor now describes a large family of proteins that feature an unusually broad ligand-binding specificity and are composed of 8 subclasses (1). The first scavenger receptor, scavenger receptor $\mathrm{AI} / \mathrm{II}$ (SR-AI/II) was identified during studies of modified lipoprotein uptake in atherosclerotic plaques (2). It was soon apparent that macrophages (as well as other cell types) bear several forms of scavenger receptors that have both similar and distinct features. The macrophage class A scavenger receptors (SRAs) bind many, but not all, polyanionic mol-

Nonstandard abbreviations used: AM, alveolar macrophage; BAL, bronchoalveolar lavage; ELF, epithelial lining fluid; MARCO, macrophage receptor with collagenous structure; MIP-2, macrophage inflammatory protein-2; PMN, polymorphonuclear leukocyte; PON-GPC, 1-palmitoyl-2-(9'-oxo-nonanoyl)-glycerophosphocholine; RNS, reactive nitrogen species; ROFA, residual oil fly ash; SR, scavenger receptor; SRA, scavenger receptor class A; SR-AI/II, scavenger receptor AI/II.

Conflict of interest: The author has declared that no conflict of interest exists.

Citation for this article: J. Clin. Invest. 117:601-604 (2007). doi:10.1172/JCI31549. ecules including acetylated or oxidized LDL, polyinosinic acid, phosphatidylserine, dextran sulfate, and components of Gram-negative and -positive bacteria (e.g., endotoxin and lipotechoic acid, respectively), prompting one expert to characterize them as "molecular flypaper" (3).

The broad recognition capabilities of scavenger receptors are especially significant to the function of alveolar macrophages (AMs), the primary pulmonary innate immunity sentinel cell. AMs are largely responsible for binding, ingestion, and ultimately clearance of numerous inhaled macromolecules, particles, and pathogens that reach the lower respiratory tract. Previous studies have focused on two SRAs expressed on AMs: the founding member, SR-AI/II, and the more recently described macrophage receptor with collagenous structure (MARCO). SR-AI/II and MARCO knockout mice show increased susceptibility to bacterial pneumonia and more robust inflammatory responses to inhaled environmental particles $(4,5)$, supporting an important role for scavenger receptors in pulmonary innate defense against exogenous challenges. One can envision an evolutionary advantage for scavenger receptor broad recognition because oxidative stress (which occurs under numerous pathologic conditions) initiates lipid oxidation, producing a plethora of reaction/ decomposition products, many of which show biological activities. Thus, if scavenger receptor-lipid binding contributes to bioactive species removal, some degree of nonspecificity in ligand recognition should augment efficacy across a spectrum of lung oxidative and inflammatory processes wherein lipid oxidation occurs.

\section{Pulmonary oxidant challenge}

Ozone and many other inhaled environmental oxidants, including particulates, produce diverse biologic effects that impact both acute and long-term public health. For obvious reasons, long-standing attention has centered on pulmonary sequelae such as physiologic impairments, inflammation, cell injury, and airway remodeling. Epidemiologic and laboratory studies indicate that environmentally relevant exposures may lead to abnormalities in lung growth and development. Importantly, both acute and chronic cardiovascular effects may also occur. In the respiratory tract, the epithelial lining fluid (ELF), which overlays all respiratory tract surfaces, is the first compartment that inhaled materials contact. The physicochemical properties of ozone (high reactivity, poor water solubility) constrain its diffusion through the ELF to the underlying epithelium, so that cellular perturbations likely result from products generated during extracellular (i.e., ELF) reactions $(6,7)$. Because the magnitude of specific biologic responses varies across species, 


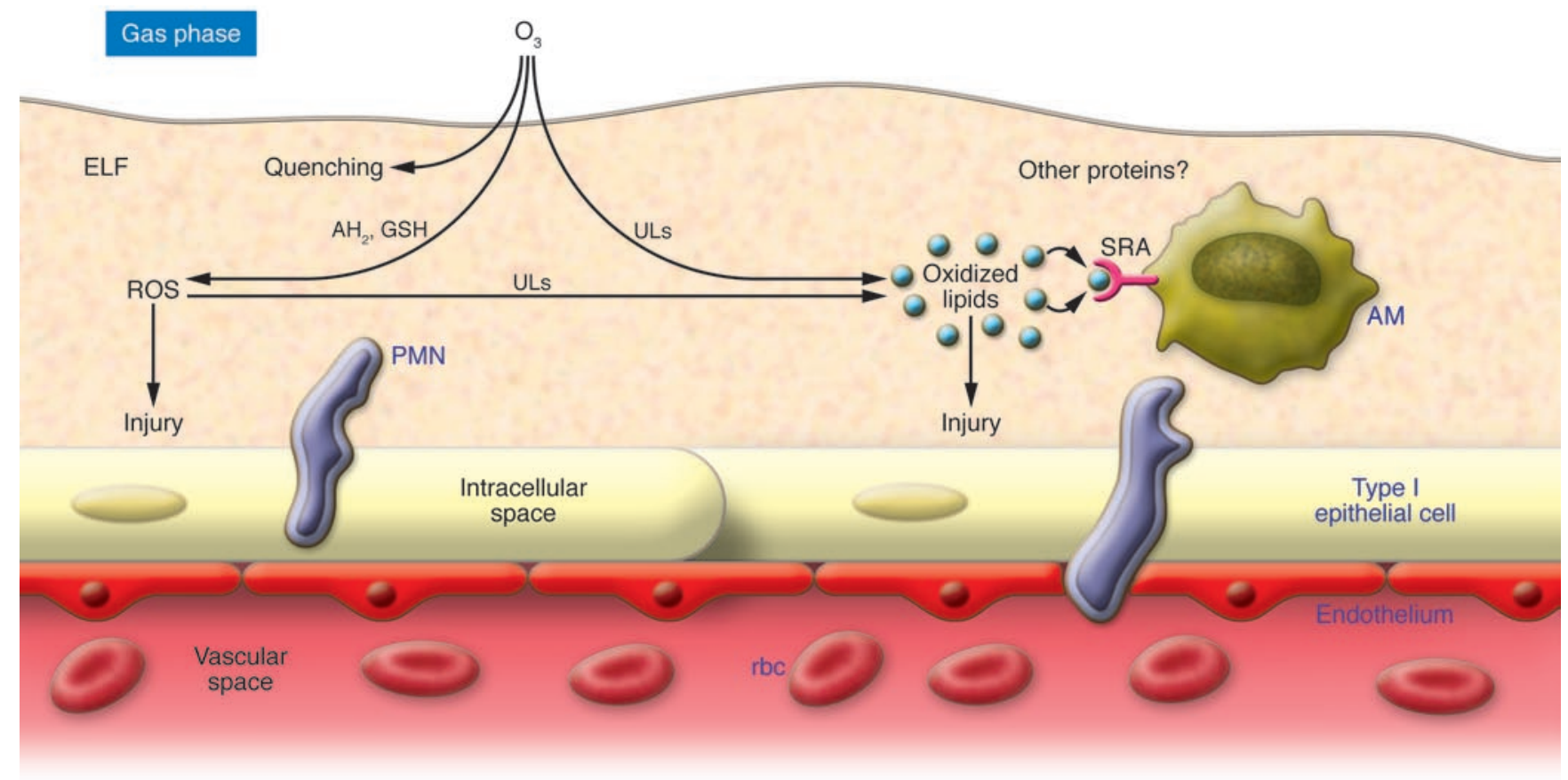

\section{Figure 1}

The lung surface is protected against inhaled oxidants. The respiratory tract surface is covered by the ELF. In this example, inhaled ozone $\left(\mathrm{O}_{3}\right)$ first dissolves in the ELF aqueous phase, followed by facile reaction with antioxidants (e.g., reduced ascorbate, $\mathrm{AH}_{2}$, and reduced glutathione, GSH), which can lead to secondary ROS production. Competing reactions between ROS and ELF unsaturated lipids (ULs) produce specific oxidized (ozonation) products. Influences of ELF protein reactions remain undefined. Due to its reactivity, it is unlikely that ozone directly contacts cellular membranes. ROS and lipid autoxidation initiated by decomposition of ozonation products lead to the generation nonspecific oxidized lipids. Secondary oxidants formed in the ELF may drive membrane unsaturated lipid oxidation, which likely contributes to the overall pool of nonspecific oxidation products. Both ROS and oxidized lipids can directly injure epithelial cells and/or initiate signaling cascades that drive PMN influx. Based on the current report from Dahl et al. (10), AM SRAs bind and internalize oxidized lipids, thereby reducing their effects on the lung epithelium. The net result limits the extent of bioactive lipid-induced inflammation. The specifics of scavenger receptor-oxidized lipid recognition and binding affinities, effects of scavenger receptor polymorphism, dispositions of internalized lipids, and composite mechanisms of how scavenger receptor-oxidized lipid binding influence PMN influx and related lung surface perturbations await further characterization.

strains, and individuals, multiple potentially independent pathways likely contribute to the overall biological outcomes. Consequently, studies that elucidate both the pathways and their governing determinants are critical to understanding the mechanisms of human susceptibility variations and disease pathogenesis.

Although ozone and residual oil fly ash (ROFA, a model of urban particulates) both induce oxidative stress, they do so by differing initial mechanisms. Ozone undergoes facile reaction with a variety of ELF constituents, most notably lowmolecular weight antioxidants and unsaturated lipids (5). Unlike most oxidants, ozone directly adds to the double bond of monounsaturated lipids. Consequently, its ELF reactions produce secondary oxidants, ozone-specific products (lipid ozonation products), and nonspecific products from direct lipid reactions and ensuing autoxidation $(8,9)$. ROFA contains an admixture of transition metals that can redox cycle within the ELF (or epithelial/AM intracellular spaces) to primarily initiate ROS and antioxidant radical production, which can subsequently lead to additional reactive species generation. Consequently, ozone generates some unique chemical species, while ROFA likely does not.

\section{Investigating the scavenger receptor-lipid connection}

In this issue of the JCI, Dahl et al. (10) used an initial discovery approach wherein genetic screening in mice elucidated that scavenger receptor expression increased during ozone exposure. While scavenger receptors are known to be related to lipid homeostasis during atherogenesis, within the lung, scavenger receptors were generally considered to primarily bind inhaled microbial agents and particulate matter, but not necessarily oxidized lipids. Because lipid products derived from both direct ozonation and ozoneinitiated autoxidation had been clearly documented in vivo and demonstrated proinflammatory (bioactive) properties, it was logical to investigate potential links between exposure-related scavenger receptor upregulation and ELF reaction products (lipid) and whether any relationships might affect exposure responses. These studies, which employed environmental and pure chemical exposures, mice with a genetic deletion of MARCO or SR-AI/II, and cell culture models, resulted in the identification of a remarkable impact of AM scavenger receptor expression on lung responses to bioactive lipids.

In these studies (10), unique ozonation product 1-palmitoyl-2-(9'-oxo-nonanoyl)glycerophosphocholine (PON-GPC) and a nonspecific autoxidation product $5 \beta, 6 \beta$ epoxycholesterol, which are produced during ozone exposure $(11,12)$, were directly instilled into wild-type as well as MARCO and SR-AI/II knockout mice. Scavenger receptors were found to affect inflammation, as measured by polymorphonuclear 
leukocyte (PMN) numbers in bronchoalveolar lavage (BAL) after exposure to both oxidation products, providing further evidence of the broad receptor binding of lipid-derived ligands. Furthermore, $\mathrm{MARCO}^{-/-}$mice were also reported to show greater neutrophilia in response to tobacco smoke challenge (data not shown in article), although because of the complexities of tobacco smoke composition (greater than 4,000 compounds, including gas-phase oxidants and particulates), the direct link to lipid binding remains unclear. Interestingly, while the presence of both MARCO and SR-AI/II suppressed PMN influx consequent to ROFA (administered as an aerosolized leachate) and direct oxidized lipid instillation, SR-AI/II was less effective in inhibiting ozoneinduced inflammation than MARCO, suggesting that although the ozone-specific product PON-GPC was recognized by both receptors, ozone exposure may have generated other ligands with lower SRA affinity than MARCO. Based on the composite data, the authors concluded that AM scavenger receptors play a critical role in decreasing pulmonary inflammation resulting from inhaled oxidant exposure by scavenging proinflammatory oxidized lipids from the lung fluid lining layer (Figure 1).

\section{Advances to the field}

The current report by Dahl et al. (10) highlights several intriguing issues. While the investigations focused on environmentally related stressors (e.g., ozone and ROFA), the results emphasize that environmental studies can reveal new insights into fundamental biologic processes and serve as unique investigational tools. In addition, these results clearly warrant consideration beyond environmental lung injury. Furthermore, these studies underscore the utility of genetic screening, as the identification of scavenger receptor upregulation during inhaled oxidant challenge would have been less likely without discovery as a first approach.

Generally, both chemical (including environmental and clinical, e.g., therapeutic $\mathrm{O}_{2}$ ) and microbial challenges in the lung lead not only to cytokine/chemokine release with associated inflammatory cell recruitment, activation, and multiple mechanisms of oxidant release, but also to upregulation of airway and parenchymal oxidant production via oxidases/peroxidases and mitochondrial perturbations.
During pulmonary challenges, rodents in particular increase production of reactive nitrogen species (RNS) via iNOS upregulation. Enhanced NO generation leads to production of nitrogen dioxide and carbonate radicals (from peroxynitrite formation with subsequent nitrosoperoxycarbonate anion homolysis), both strong oxidants capable of initiating lipid oxidation. However, because protein nitration occurs in numerous lung pathology states (e.g., acute respiratory distress syndrome), humans clearly undergo similar RNS chemistry (13). Unsaturated lipids are necessary for cell membrane function and are secreted as part of the surfactant and mucus complexes, where they generally constitute greater than $20 \%$ of the ELF lipid pool and are therefore present at a sufficient concentration to generate appreciable oxidation product yields. However, because ELF reactant profiles (antioxidants, unsaturated lipids, etc.) show considerable variability across species, anatomic sites, and disease states, the competition reaction kinetics among oxidants and the multiple ELF substrates will dictate the prevalence of reaction pathways and thus specific product formation.

One minor caveat to the authors' overall conclusions (10) involves the fact that although direct oxidized lipid application in general recapitulated the link between scavenger receptors and ozone/ROFA exposure-mediated inflammation, it remains equivocal as to whether these lipids accounted for the only receptor-ligand interactions modulating cytokine production, inflammation, and protein movement into the ELF (permeability). Thus, other chemical moieties (e.g., peptide fragments and posttranslationally modified proteins) could play important roles, but would have been difficult to distinguish during the current ozone/ROFA in vivo experimental approaches. Regardless, the current study underscores the importance of scavenger receptor expression in lung inflammation, especially with regard to the fact that scavenger receptors modulate oxidized lipid bioactivites. In this case, AMs appeared to inhibit inflammation rather than initiate and/or amplify PMN recruitment. Furthermore, subtle differences in AM ligand binding and/or receptor activity kinetics could influence downstream events and account for some of the diversity observed relative to species, strain, and individual susceptibility to oxidant insult. Consequently, it will be important not only to determine how scavenger receptor expression correlates to human disease states, but also to more thoroughly characterize which oxidized lipids serve as human AM scavenger receptor ligands and how regulating oxidized lipid homeostasis modulates lung inflammatory processes, including disease progression, in which AM play key roles.

\section{Curiosities and considerations for future directions}

One simple question involves why scavenger receptor genetic deletion produced notable increases in PMN recruitment to the lung surface, especially if AMs were the primary affected cell type. Ozone generates focal lesions that occur predominately in the conducting airways (14) and bronchoalveolar junctions. Most AMs encounter little or no ozone because inhaled ozone is removed by conducting airway wall reactions during proximal-to-distal longitudinal transport. However, ozone was administered continuously for at least 24 hours, which may have induced more diffuse effects and/or led to alterations in ELF unsaturated lipid homeostasis, thereby affecting precursor lipid availability. Aerosolized ROFA leachate and the instilled lipids were likely distributed throughout the lung, so that even with the shorter exposure and/or sampling times, the effects may have been more anatomically widespread. This is suggested by the data illustrating that the oxidized lipids did not upregulate macrophage inflammatory protein-2 (MIP-2) or increase inflammation in the scavenger receptor-competent animals (10). It would be interesting to determine whether shorter ozone exposure times or analyses specific to the conducting airways would demonstrate equivalent effects with scavenger receptor genetic deletion.

Although it was reasonable to conclude that inhibiting PMN influx was attributable to scavenger receptor-mediated bioactive lipid removal, this would implicitly require that oxidized lipid removal rates exceeded their generation, effectively reducing bioactive lipid concentrations below critical thresholds. Furthermore, AMs would need to be localized near sites of oxidized lipid generation and/or diffusive or mucociliary transport. If bioactive lipid concentrations were low, then scavenger receptor-mediated clearance would not be a substantial challenge. However, because many inhaled agents initiate oxidant stress, which is further amplified by inflammation, a substantial total pool of lipid products might be 
achieved. This should also hold true for isoprostanes. Isoprostanes are not generated directly by ozone reaction; rather, they are caused by arachadonic acid autoxidation. The isoprostane measures reported in the current study (10) represented net accumulation, not total yields or production rates. Even though scavenger receptor activity limited isoprostane accumulation, it remains equivocal whether this resulted from scavenger receptor-mediated clearance or reduced formation rates due to secondary AM-mediated effects on ROS/RNS production. The in vitro studies of AM lipid loading provided very useful initial insights, but further studies - to quantify oxidized lipid uptake versus degradation and the mass balance of generation versus removal - are needed to determine why scavenger receptor genetic deletion reduced intracellular lipid content and augmented inflammation. Furthermore, although inflammation is a hallmark ozone response, other biologic responses are not necessarily coupled and/or regulated via the same biologic pathways. It will be key in future studies to evaluate whether scavenger receptor expression specifically governs airway epithelial injury and influences other AM functions that modulate the observed responses. A critical question remains as to whether scavenger receptors contribute to suppressing inflammation by clearing ozone-generated proinflammatory lipids (15), because many of the biological processes associated with cell injury and oxidative stress tend to potentiate lipid oxidation.

It will be interesting to see how scavenger receptor receptor activity influences more environmentally relevant exposure regimens and/or other stressors. Although it was beyond the scope of this initial study, it is not entirely clear how the chosen biologic readouts (i.e., levels of BAL neutrophils, protein, isoprostanes, and MIP-2) relate to what constitutes oxidant "susceptibility” versus "resistance." Ultimately, what constitutes susceptibility versus resistance should be placed in the context of more specific measures of epithelial perturbation and injury. The contributions of cell-cell interactions and potential confounding influences of other cell types also warrant elucidation. In addition, other bioactive lipids (e.g., nitrolipids; refs. 16, 17) as well as electrophiles, operating through the electrophilic response element, have important and robust influences on signaling pathways. PMNs tend to amplify injury, but are critical for normal epithelial repair (18). If scavenger receptor activities vary, influence on PMN trafficking could have notable effects on airway repair. Consequently, the long-term effects of diminished scavenger receptor activity on repair and airway remodeling also represent important areas of future investigation. These current and future studies have substantial potential relevance to human biology. MARCO is expressed and functions on human AMs (19), and polymorphisms in both SR-AI/II and MARCO exist. Hence, further animal studies, linked to translational efforts, hold substantial promise for identifying the mechanisms responsible for known variations in human susceptibility to a wide variety of oxidative challenges.

Address correspondence to: Edward M. Postlethwait, Department of Environmental Health Sciences, School of Public Health, University of Alabama at Birmingham, 530 Ryals Building, 1530 3rd Ave. South, Birmingham, Alabama 352940022, USA. Phone: (205) 934-7085; Fax: (205) 975-6341; E-mail: EPostlethwait@ ms.soph.uab.edu.

1. Murphy, J.E., Tedbury, P.R., Homer-Vanniasinkam, S., Walker, J.H., and Ponnambalam, S. 2005. Biochemistry and cell biology of mammalian scavenger receptors. Atherosclerosis. 182:1-15.

2. Goldstein, J.L., Ho, Y.K., Basu, S.K., and Brown, M.S. 1979. Binding site on macrophages that mediates uptake and degradation of acetylated low density lipoprotein, producing massive cholesterol deposition. Proc. Natl. Acad. Sci. U. S. A. 76:333-337.

3. Krieger, M. 1992. Molecular flypaper and atherosclerosis: structure of the macrophage scavenger receptor. Trends Biochem. Sci. 17:141-146.

4. Arredouani, M., et al. 2004. The scavenger receptor
MARCO is required for lung defense against pneumococcal pneumonia and inhaled particles. J. Exp. Med. 200:267-272.

5. Arredouani, M.S., et al. 2006. The macrophage scavenger receptor SR-AI/II and lung defense against pneumococci and particles. Am. J. Respir. Cell Mol. Biol. 35:474-478.

6. Postlethwait, E.M., Cueto, R., Velsor, L.W., and Pryor, W.A. 1998. $\mathrm{O}_{3}$-induced formation of bioactive lipids: estimated surface concentrations and lining layer effects. Am. J. Physiol. Lung Cell. Mol. Physiol. 274:L1006-L1016.

7. Ballinger, C.A., et al. 2005. Antioxidant-mediated augmentation of ozone-induced membrane oxidation. Free Radic. Biol. Med. 38:515-526.

8. Cueto, R., Squadrito, G.L., and Pryor, W.A. 1994. Quantifying aldehydes and distinguishing aldehydic product profiles from autoxidation and ozonation of unsaturated fatty acids. Meth. Enzymol. 233:174-182.

9. Pryor, W.A., Bermudez, E., Cueto, R., and Squadrito, G.L. 1996. Detection of aldehydes in bronchoalveolar lavage of rats exposed to ozone. Fundam. Appl. Toxicol. 34:148-156.

10. Dahl, M., et al. 2007. Protection against inhaled oxidants through scavenging of oxidized lipids by macrophage receptors MARCO and SR-AI/II. J. Clin. Invest. 117:757-764. doi:10.1172/JCI29968.

11. Uhlson, C., et al. 2002. Oxidized phospholipids derived from ozone-treated lung surfactant extract reduce macrophage and epithelial cell viability. Chem. Res. Toxicol. 15:896-906.

12. Pulfer, M.K., Taube, C., Gelfand, E., and Murphy, R.C. 2005. Ozone exposure in vivo and formation of biologically active oxysterols in the lung.J. Pharmacol. Exp. Therap. 312:256-264.

13. Sittipunt, C., et al. 2001. Nitric oxide and nitrotyrosine in the lungs of patients with acute respiratory distress syndrome. Am. J. Respir. Crit. Care Med. 163:503-510.

14. Postlethwait, E.M., et al. 2000. Three-dimensional mapping of ozone-induced acute cytotoxicity in tracheobronchial airways of isolated perfused rat lung. Am. J. Respir. Cell Mol. Biol. 22:191-199.

15. Kafoury, R.M., et al. 1999. Induction of inflammatory mediators in human airway epithelial cells by lipid ozonation products. Am. J. Respir. Crit. Care Med. 160:1934-1942.

16. Cui, T., et al. 2006. Nitrated fatty acids: endogenous anti-inflammatory signaling mediators. J. Biol. Chem. 281:35686-35698.

17. Wright, M.M., et al. 2006. Fatty acid transduction of nitric oxide signaling: nitrolinoleic acid potently activates endothelial heme oxygenase 1 expression. Proc. Natl. Acad. Sci. U. S. A. 103:4299-4304.

18. Hyde, D.M., et al. 1999. Neutrophils enhance clearance of necrotic epithelial cells in ozone-induced lung injury in rhesus monkeys. Am. J. Physiol. 277:L1190-L1198.

19. Arredouani, M.S., et al. 2005. MARCO is the major binding receptor for unopsonized particles and bacteria on human alveolar macrophages. J. Immunol. 175:6058-6064. 